\title{
Dynamic Bayesian networks and variable length genetic algorithm for designing cue- based model for dialogue act recognition
}

\begin{abstract}
The automatic recognition of dialogue act is a task of crucial importance for the processing of natural language dialogue at discourse level. It is also one of the most challenging problems as most often the dialogue act is not expressed directly in speaker's utterance. In this paper, a new cue-based model for dialogue act recognition is presented. The model is, essentially, a dynamic Bayesian network induced from manually annotated dialogue corpus via dynamic Bayesian machine learning algorithms. Furthermore, the dynamic Bayesian network's random variables are constituted from sets of lexical cues selected automatically by means of a variable length genetic algorithm, developed specifically for this purpose. To evaluate the proposed approaches of design, three stages of experiments have been conducted. In the initial stage, the dynamic Bayesian network model is constructed using sets of lexical cues selected manually from the dialogue corpus. The model is evaluated against two previously proposed models and the results confirm the potentiality of dynamic Bayesian networks for dialogue act recognition. In the second stage, the developed variable length genetic algorithm is used to select different sets of lexical cues to constitute the dynamic Bayesian networks' random variables. The developed approach is evaluated against some of the previously used ranking approaches and the results provide experimental evidences on its ability to avoid the drawbacks of the ranking approaches. In the third stage, the dynamic Bayesian networks model is constructed using random variables constituted from the sets of lexical cues generated in the second stage and the results confirm the effectiveness of the proposed approaches for designing dialogue act recognition model.
\end{abstract}

